\title{
Annual incidence
}

INSERM

\section{Source}

INSERM. (1999). Orphanet: an online rare disease and orphan drug data base. annual incidence. ORPHA:409967

Number of newly diagnosed cases in a population in 1 year. 